\title{
Cable-Stiffened Pantographic Deployable Structures Part 2: Mesh Reflector
}

\author{
Z. You*and S. Pellegrino ${ }^{\dagger}$ \\ University of Cambridge, Cambridge CB2 1PZ, England, United Kingdom
}

\begin{abstract}
The general concept of deployable structures based on pantographs that are deployed and stiffened by means of cables is applied to the design of the support structure for a large mesh reflector. The two main components of this structure are a cable-stiffened pantographic ring that deploys and pretensions a cable network that, in turn, provides a series of stiff, geometrically accurate support points to which a reflective wire mesh or flexible membrane would be connected. The pantographic ring is a highly redundant structure with an internal mechanism that permits synchronous deployment without any strain in the rods. The geometric conditions that have to be satisfied in order for an $n$-sided ring to fold without any strain are investigated, including the effects of joint size. An experimental model has been designed and tested. In the folded configuration, it has a diameter of $0.6 \mathrm{~m}$ and height of $1.2 \mathrm{~m}$; in the deployed configuration, it has a diameter of $3.5 \mathrm{~m}$. Stiffness and deployment tests on this model have shown its behavior to be linear and the maximum shape error to be $\pm^{0.3} \mathbf{~ m m}$.
\end{abstract}

\section{Introduction}

T HIS paper is the second in a series that deals with a new type of deployable structures where a foldable bar structure, which consists of pairs of straight bars connected by pivots and forming a pantograph, is deployed and stiffened by two sets of cables, known as active and passive cables. The passive cables are short cable elements connected to joints of the pantograph that get farther apart during deployment, and the length of each passive cable is such that it becomes taut when the pantograph is fully deployed. The active cables are longer elements that run over small pulleys and whose overall length is controlled by one or more electric motors. The layout of these cables is such that deployment of the pantograph can be activated by shortening the length of at least one of the active cables. Once the pantograph is deployed, and hence all of the passive cables are taut, it is possible to set up an overall state of prestress in the structure such that all cables are in a state of tension.

The first paper in this series showed how this concept can be applied to deployable mast structures, using as a particular example a straight mast based on a pantograph consisting of a stack of identical modules with triangular cross section. ${ }^{1}$ The present paper shows an application of the same general approach to the design of the support structure for a large deployable mesh reflector (DMR). Large deployable antennas of this type are required for high-gain telecommunication, remote sensing, and scientific missions. ${ }^{2}$

General reviews of deployable reflectors have been compiled by Freeland $^{3}$ and, more recently, by Roederer and Rahmat-Sahmii. ${ }^{4}$ Broadly speaking, there are two types of reflectors: solid-surface reflectors, where the reflective surface is a continuous dish, and mesh reflectors, where the reflective surface consists of interlaced, electrically conductive thin wires. The second type is suitable only for transmission of low-frequency waves. From a structural viewpoint, standard requirements for deployable reflectors include: low mass, compact packaged volume, simple and reliable deployment mechanisms, and sufficient stiffness to avoid dynamic coupling between the reflector and the spacecraft attitude control system. A final requirement, specific to reflector structures, is that the shape error of the reflective surface, i.e., its deviation from the best-fit paraboloid, should be within a specified limit, which is related to the shortest wavelength to be transmitted. Typically, a surface accuracy in the range $1-10 \mathrm{~mm}$ is required.

Received Feb. 1, 1996; revision received March 24, 1997; accepted for publication May 5, 1997. Copyright 997 by S. Pellegrino and Z. You. Published by the American Institute deronautics and Astronautics, Inc., with permission.

*Research Fellow, Department of Engineering, TrumpingtonStreet. Member AIAA.

${ }^{\dagger}$ Lecturer in Structural Engineering, Department of Engineering, Trumpington Street. Member AIAA.
Two existing concepts that are related to the new concept presented are the hoop/column antenna and the tension truss antenna. The hoop/column antenna, ${ }^{5,6}$ developed by the Harris Corporation in the 1980s, consists of a telescopic column that deploys from a central hub and a deployable hoop, made of articulating segments, that is supported by cables connected to the lower and upper column extremities, at one end, and to hoop joints at the other end. The reflective mesh, attached both to the hoop and to the hub, is shaped by a series of catenary cord elements, which support and contour the reflective mesh surface. Deployment of this antenna is accomplished in three steps: column extension, hoop/surface deployment, and surface prestress.

The tension truss antenna, developed by Miura and Miyazaki, is based on a kinematically determinate cable net forming a coarse triangular network, pretensioned against a backup structure by soft tie cords. The backup structure consists of six radially arranged deployable masts and a centralmast, all originating from a rigid hub, which are deployed simultaneously. A reflective mesh is attached to the cable network.

The DMR has conceptual similarities to both of these deployable reflectors. It has a deployable edge frame, like the hoop/column antenna, and a kinematically determinate cable network, like the tension truss antenna; however, both the edge frame and the cable network are of a new type. Deployment and prestressing of the DMR are controlled by only two dc motors and are completed in one step. Furthermore, the new concept is not tied to a particular type of subreflector/antenna feed support system, because it does not have a central mast. Hence, different options can be considered, depending on mission requirements.

The deploymentsequence of a model structure based on the DMR concept is shown in Fig. 1. The cable-stiffenedpantographicdeployable frame of toroidal shape deploys and pretensionsa cable network spanningthe region within the frame. The cable net, in turn, provides a series of stiff, geometrically accurate support points to which a reflective wire mesh or flexible membrane would be connected, thus forming a geometrically accurate surface that closely approximates a paraboloid.

The layout of this paper is as follows. Section II introduces the cable-stiffened pantographic ring that forms the edge frame of the DMR. Section III introduces two types of cable networks that can be connected to the deployable ring. The design and testing of an experimental model are presented in Secs. IV and V. Section VI concludes the paper.

\section{Deployable Edge Frame}

Following Ref. 1, the deployable edge frame is based on the concept of cable-stiffenedpantographs. Hence, first we will identify 

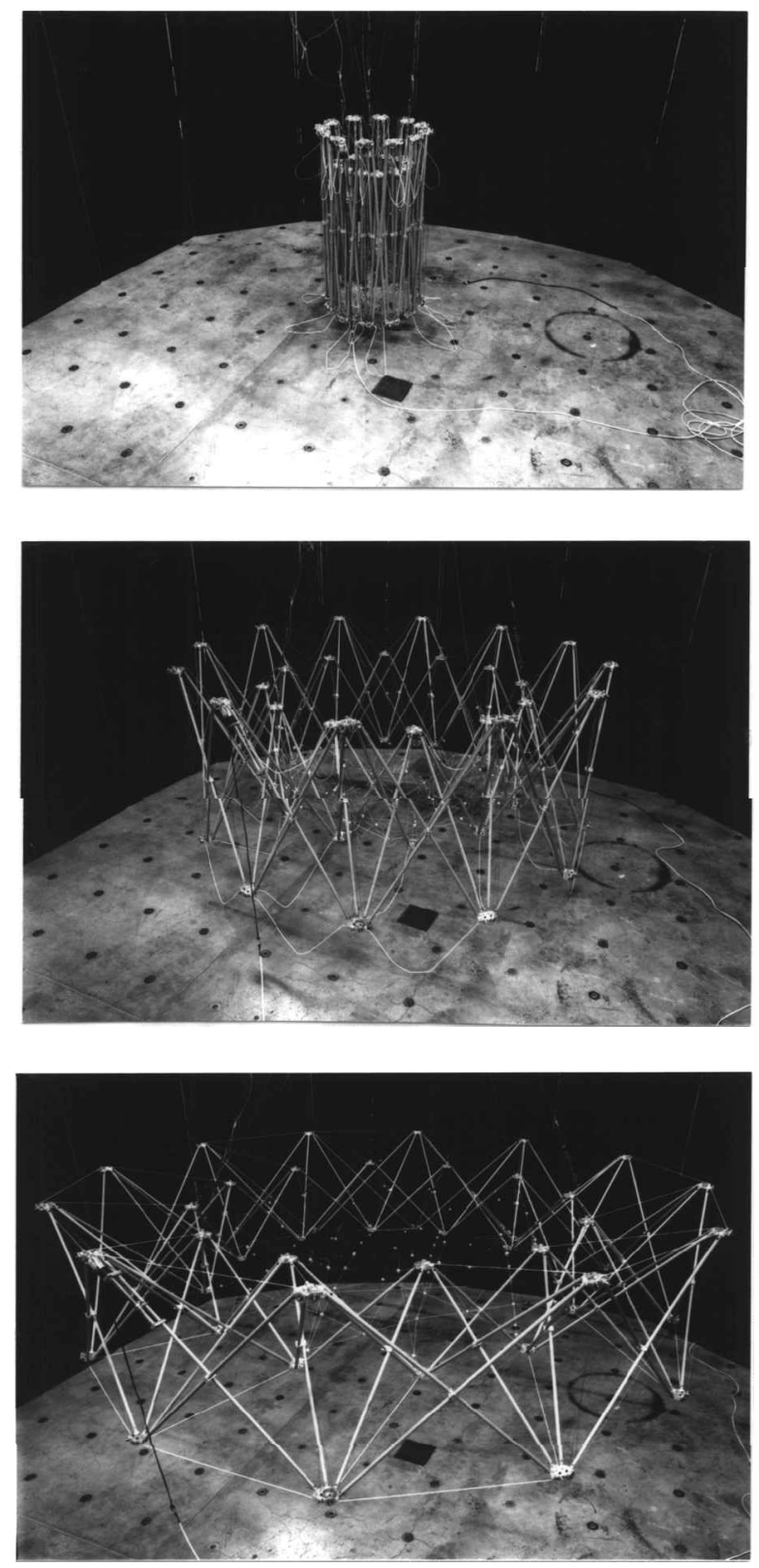

Fig. 1 Deployment sequence of DMR model.

a suitable foldable pantographstructure, and then we will investigate possible arrangements of the active and passive cables.

\section{A. Ring Pantograph}

Only a small number of ringlike pantographs are known that can be folded without straining any members ${ }^{8}$; the particular solution that is of interest makes use of pairs of identical straight rods of length $2 \ell, \ell+L$, and $2 L$, connected by scissor hinges. In two of these pairs of rods (Figs. 2a and 2c), the hinge is located halfway between the end connectors, whereas in the third pair (Fig. 2b), the hinge is at a distance $\ell$ from one end and at a distance $L$ from the other end.

Assuming, initially, that the joint eccentricities can be neglected, i.e., $\lambda=\Lambda=0$, these three pairs of rods can be connected in the sequencea, $b, c$ to form a simple two-dimensionalfoldable structure. If the deployment angle $\theta$ is the same for all rod pairs, the righthand-side connectors of pair a are $2 \ell \cos (\theta / 2)$ apart and can be connected with the corresponding left-hand-sideconnectors of pair $\mathrm{b}$, also $2 \ell \cos (\theta / 2)$ apart. If these connectors are linked together, a four-bar chain is formed whose bars are all of length $\ell$. This chain fits togetherfor all values of $\theta$, without straining any member. Similarly, the right-hand-side connectors of pair b fit with the left-hand-side connectors of pair c for all values of $\theta$ and, hence, they can be

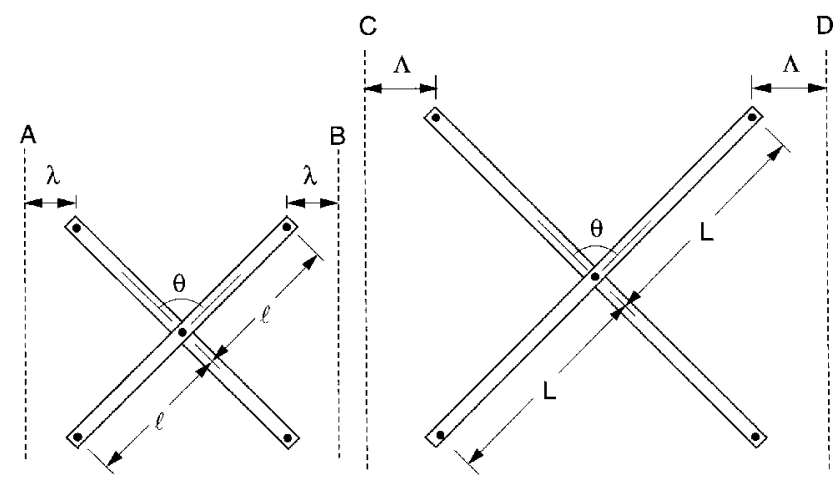

a) Inner rod pair

c) Outer rod pair

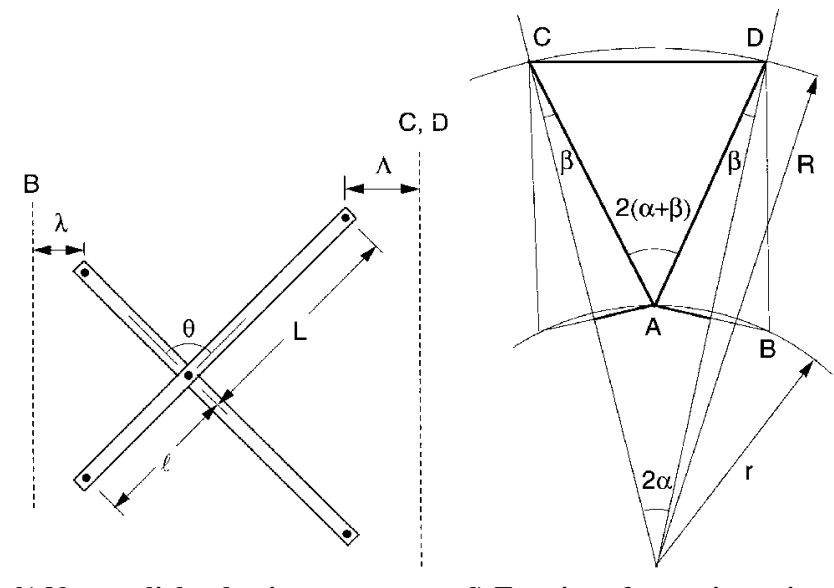

b) Near-radial rod pair

d) Top view of repeating unit

Fig. 2 Elements of ring pantographs.

linked to form a four-bar chain whose bars are all of length $L$. These considerations are also valid if the three pairs of rods are not in the same plane, and they can also be extended to any number of rod pairs. The key condition that must be satisfied to be able to fold this pantograph without straining its members is that the semilengths of the rods in each four-bar chain must all be equal to either $\ell$ or $L$, which implies that the angle between rods in each pair is $\theta$. This folding condition is a special, almost trivial case of a general condition derived by Escrig. ${ }^{9}$

A ringlike pantograph can be formed by connecting several pairs of rods of the type shown in Figs. 2a-2c. However, in addition to satisfying a series of local compatibility conditions, one for each four-barchain, it is also necessary to satisfy the overall compatibility condition that the whole ring should fit together for all values of $\theta$. In general, the simplest way of satisfying the latter condition is to design pantograph structures that consist of $n$ identical modules and to design the repeating module so that it maintains a constant angle of embrace regardless of the value of $\theta$.

The pantograph used in the DMR (Fig. 1) is formed from $n=12$ pairs of type a rods forming an inner ring and $n=12$ pairs of rods of type c forming an outer ring. These two rings are connected by $2 n$ pairs of rods of type $b$. A top view of the basic module of a general $n$-sided pantograph is shown in Fig. $2 \mathrm{~d}$, where $A B$ and $C D$ are, respectively, a pair of rods of type a and of type c, seen from the top, whereas $A C$ and $A D$ are rods of type b. Next, it will be shown that for any value of $n$ there is a unique ratio $\ell / L$ for which the pantograph satisfies the overall compatibility condition just described.

An expression for the angle $\alpha$, which must be a constant, can be obtained as follows. The projected rod lengths, shown in Fig. 2d, have the following expressions:

$$
\begin{gathered}
\overline{A B}=2 \overline{A D} \sin \beta \\
\overline{C D}=2 \overline{A D} \sin (\alpha+\beta)
\end{gathered}
$$


Expressing $\overline{A B}, \overline{A D}$, and $\overline{C D}$ in terms of $\ell, L$, and $\theta$

$$
\begin{gathered}
\overline{A B}=2 \ell \sin (\theta / 2) \\
\overline{A D}=(\ell+L) \sin (\theta / 2) \\
\overline{C D}=2 L \sin (\theta / 2)
\end{gathered}
$$

Substituting Eqs. (3) and (4) into Eq. (1), and Eqs. (4) and (5) into Eq. (2) and dividing by $\sin (\theta / 2)$ gives the following two equations:

$$
\begin{gathered}
(\ell+L) \sin \beta=\ell \\
(\ell+L) \sin (\alpha+\beta)=L
\end{gathered}
$$

From Eq. (6)

$$
\beta=\arcsin [\ell /(\ell+L)]
$$

Dividing the left- and the right-hand sides of Eq. (6) by the corresponding sides of Eq. (7) gives, after simple manipulations,

$$
\frac{L}{\ell}=\sin \alpha \sqrt{\frac{1}{\sin ^{2} \beta}-1}+\cos \alpha
$$

Substituting Eq. (8) for $\beta$ and rearranging gives the following quadratic equation in $L / \ell$ :

$$
\cos ^{2} \alpha(L / \ell)^{2}-2\left(\cos \alpha+\sin ^{2} \alpha\right)(L / \ell)+\cos ^{2} \alpha=0
$$

whose solutions are

$$
\frac{L}{\ell}=\frac{\cos \alpha+\sin ^{2} \alpha+\sin \alpha \sqrt{1+2 \cos \alpha}}{\cos ^{2} \alpha \sqrt{ }}
$$

It can be shown that these two solutions are reciprocalof one another. Hence, if the positive sign is taken in Eq. (11), the inner rings of the corresponding pantograph is formed by pairs of rods of type a, whereas the outer ring is formed by rods of type c. If the negative sign is taken, the type of rod pairs are exchanged. Therefore, it is pointless to consider both solutions given in Eq. (11).

In conclusion, the geometric properties of all foldable ring pantographs that form closed chains of triangular prisms with $n$-fold rotational symmetry and that consist of three different rod pairs only are defined as follows:

$$
\frac{L}{\ell}=\frac{\cos \alpha+\sin ^{2} \alpha+\sin \alpha \sqrt{1+2 \cos \alpha}}{\cos ^{2} \alpha \sqrt{ }}
$$

where $\alpha=360 \mathrm{deg} / 2 n$. This expression has been plotted in Fig. 3 .

The physical size of the joints of the pantograph has not been considered so far, hence, the preceding final result is valid only if the axes of all connectorson the same joint assembly can be arranged to meet at a single point, which is not only difficult to achieve at the inner joints of the pantograph, whose rods form an angle greaterthan $180 \mathrm{deg}$, but may also restrict the deployment process. Therefore, the preceding analysisneeds to be extended to include joints of finite size.

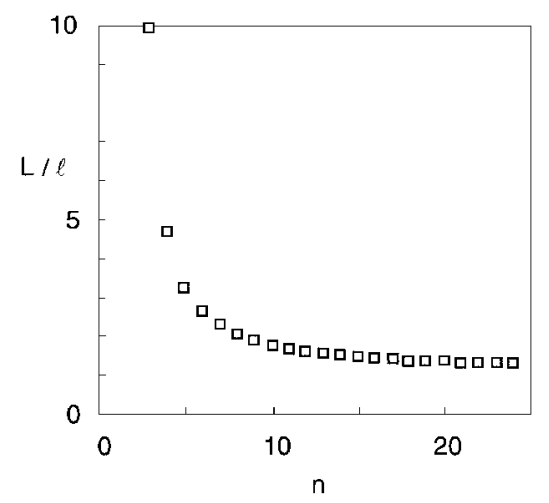

Fig. 3 Relationship between rod lengths and number of sides.
For simplicity, it will be assumed that all connectors on the same joint assembly are coplanar and equidistant from the center of the joint and that there are only two different types of joints, whose eccentricities are, respectively, $\lambda$ and $\Lambda$ for joints lying on the inner ring and on the outer ring. Equations (3-5) are modified as follows:

$$
\begin{gathered}
\overline{A B}=2 \ell \sin (\theta / 2)+2 \lambda \\
\overline{A D}=(\ell+L) \sin (\theta / 2)+(\lambda+\Lambda) \\
\overline{C D}=2 L \sin (\theta / 2)+2 \Lambda
\end{gathered}
$$

Substituting Eqs. (13) and (14) into Eq. (1) and Eqs. (14) and (15) into Eq. (2) and dividing by 2 gives

$$
\ell \sin (\theta / 2)+\lambda=(\ell+L) \sin (\theta / 2) \sin \beta+(\lambda+\Lambda) \sin \beta
$$

$L \sin (\theta / 2)+\Lambda=(\ell+L) \sin (\theta / 2) \sin (\alpha+\beta)+(\lambda+\Lambda) \sin (\alpha+\beta)$

This system of equations is a more complex version of the system formed by Eqs. (6) and (7). Instead of looking for a general solution, we are interested in the particular solution that makes the first term on the right-hand side of each equation equal to the corresponding term on the left-hand side and, also, the second term equal to the corresponding second term. Hence, instead of solving Eq. (16), we shall solve the following system:

$$
\begin{gathered}
\ell \sin (\theta / 2)=(\ell+L) \sin (\theta / 2) \sin \beta \\
L \sin (\theta / 2)=(\ell+L) \sin (\theta / 2) \sin (\alpha+\beta) \\
\lambda=(\lambda+\Lambda) \sin \beta \\
\Lambda=(\lambda+\Lambda) \sin (\alpha+\beta)
\end{gathered}
$$

The first two equations of this system coincide with Eqs. (6) and (7), and their solution is given by Eqs. (8) and (12). The third and fourth equations of Eq. (17) are analogous to the first two, and hence their solution is

$$
\begin{gathered}
\beta=\arcsin [\lambda(\lambda+\Lambda)] \\
\frac{\Lambda}{\lambda}=\frac{\cos \alpha+\sin ^{2} \alpha+\sin \alpha \overline{1+2 \cos \alpha}}{\cos ^{2} \alpha \sqrt{1+\operatorname{an}}}
\end{gathered}
$$

Equations (8), (12), (18), and (19) are consistent for

$$
\Lambda / \lambda=L / \ell
$$

In conclusion, the geometric design of $n$-sided foldable ring pantographs of the type used in the DMR is governed by Eqs. (8), (12), and (20). For $n=12(\alpha=15 \mathrm{deg})$, these equations give

$$
\beta=22.8 \mathrm{deg} \quad \text { and } \quad(L / \ell)=(\Lambda / \lambda)=1.5821
$$

\section{B. Deployment and Stiffening by Means of Cables}

Active and passive cables are used to deploy the ring pantograph and to increase its stiffness when it is fully deployed. First, we will establish how many cables are required. The best strategy to transform a flexible pantograph into a stiff structure is to model the pantograph as a pin-jointed truss, thus neglecting the scissor hinge in each pair of rods, and to rigidize this truss by adding a series of passive cables to it. Normally, a cable is consideredas a tension only element but, because in this application a state of pretension will be applied to all cables, it can be assumed that each cable behaves as a pin-jointed bar. This assumes, of course, that the pretension magnitude is higher than any compression induced by the applied loads.

For a ring pantograph with $n=12$ sides, the truss model has 48 pin joints and 96 pin-jointed bars. The total number of cables required to make this into a rigid structure can be estimated from the generalized Maxwell's rule ${ }^{10}$

$$
3 j-b=m-s
$$

where $j$ is the number of joints, $b$ the number of bars, $m$ the number of independent inextensionalmechanisms, and $s$ the number of independent states of self-stress. Here $j=48, m \geq 6$ because the 
structure has at least six degrees of freedom as a rigid body in three dimensions, and $s>1$ so that the passive cables may be pretensioned. Substitutingthese values into Eq. (22) yields $b=139$ and, because the truss already has 96 bars, no fewer than 43 cables need to be introduced, e.g., 42 passive cables and 1 active cable.

It is desirable that the passive cables should be arranged in a symmetric way, to obtain uniform structural properties. Figure 4 shows 3 rotationally symmetric ways of introducing 12 passive cables in a 12-sided ring. In Fig. 4a the cables form an outer hoop at the top or at the bottom of the ring, whereas in Fig. $4 \mathrm{~b}$ they form an innerhoop. In Fig. $4 c$ they are arranged in near-radial directions, in a clockwise sense, and of course they can also be arranged in an anticlockwise sense, at the top or at the bottom of the ring. Thus, there are 8 different ways of arranging a set of 12 passive cables, giving a maximum of 96 passive cables. To find a suitable set of passive cables, first we select four of these eight sets and take out six cables. Then, we look for an active cable that can impose a state of pretension onto all of the passive cables. This is an iterative process, carried out with the aid of suitable computer software for the analysis of the static and kinematic properties of pin-jointed assemblies. ${ }^{11-13}$

It has been found that a good choice for the passive cables consists of two near-radial sets of cables, of the type shown in Fig. 4c, e.g., the clockwise set at the top of the pantograph and the anticlockwise set at the bottom, plus the two outer hoops shown in Fig. 4a, but without three cables in each hoop. The overall layout of these 42 cables is shown in Fig. 5a. An analysis of the equilibriummatrix for a a)

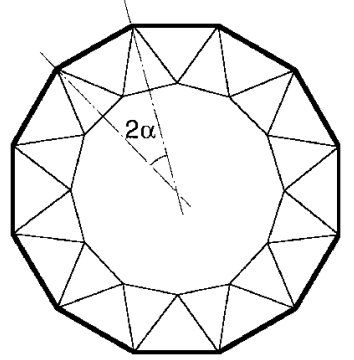

b)

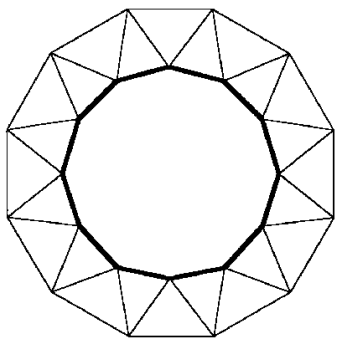

c)

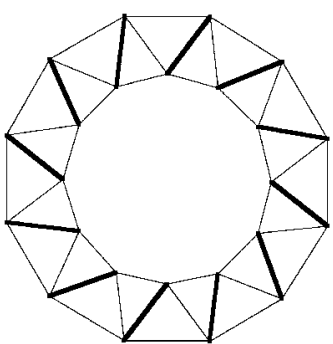

Fig. 4 Possible arrangements of passive cables, top view.

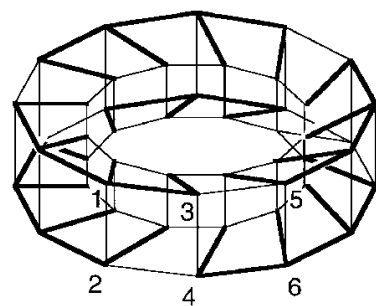

a) Full set of passive cables

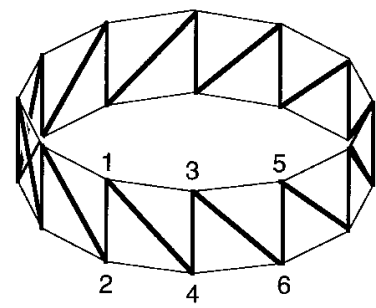

b) Active cable 1

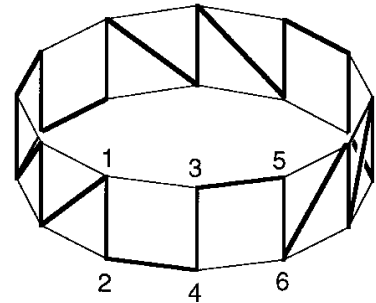

c) Active cable 2

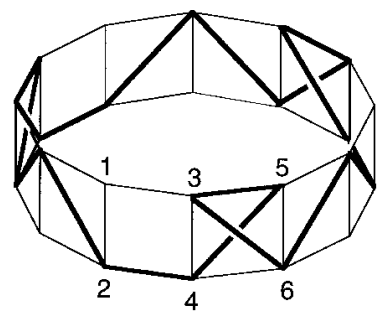

d) Active cable 3
Fig. 5 Passive and active cables. pin-jointed assembly consisting of 96 pin-jointed bars, representing the rods of the pantograph, and 42 additional bars, representing the passive cables, shows that this assembly has $m=7$ and $s=$ 1 , instead of the expected $m=6$ and $s=0$. The state of selfstress involves only the pantograph and, hence, it has no effect on the passive cables. The accompanying internal mechanism is not activated by the forces applied by the cable networks discussed in Sec. III. Furthermore, the presence of scissor hinges in the real structure will stiffen this deformation mode (see Sec. V.A).

The search for a single active cable that is capable of deploying the pantograph and also of imposing a state of uniform pretension onto all of the passive cables just selected has been unsuccessful. However, two partial solutions have been identified, which can be combined to obtain the required prestress. The first active cable is shown in Fig. 5b. It is connected to joint 1 and runs alongside rod 1-4; at joint 4 it loops over a pulley whose axis of rotation is perpendicular to a facet of the ring (1-2-3-4), then at joint 3 it goes over a pulley with axis perpendicular to the next facet of the ring (3-4-5-6), and so on until joint 1 is reached. A unit tension in this active cable is in equilibrium with uniform tensions of 0.662 in the 24 near-radial passive cables and zero tension in the remaining 18 passive cables. Axial forces of suitable magnitude in the rods of the pantograph complete this state of self-stress, but their values are not important in this analysis. The second active cable is shown in Fig. 5c. Its route includes the six gaps left in the two passive cable hoops. A unit tension in this active cable is in equilibrium with a uniform tension of 1 in the 18 passive cables that had been left unstressed by the first active cable and with a uniform compression of 0.662 in the 24 near-radial cables.

Now, a uniform pretension of all passive cables can be obtained by using both active cables. The ratio between the tensions $t_{1}$ and $t_{2}$, respectively, in active cables 1 and 2 has to be such that the tensions in the near-radial passive cables should be equal to the tension in the remaining cables. Hence,

$$
0.662 t_{1}-0.662 t_{2}=t_{2}
$$

which gives

$$
\frac{t_{1}}{t_{2}}=\frac{1+0.662}{0.662}=2.551
$$

This solution is idealin terms of the state of prestress that is achieved, but it is not ideal from the viewpoint of deployment control because the lengths of the two active cables, $L_{1}$ and $L_{2}$, respectively, need to vary in a nonproportionalfashion during deployment:

$$
\begin{gathered}
L_{1}(\theta)=24 L+24 L \cos (\theta / 2) \\
L_{2}(\theta)=12 L+24 L \cos (\theta / 2)+12 L \sin (\theta / 2)
\end{gathered}
$$

These two functionsare plotted in Fig. 6. As the pantographdeploys, $\theta$ changes from approximately 0 deg to approximately $90 \mathrm{deg}$ and $L_{1}$ decreases all of the time. Hence, deploymentcan be controlled by winding in this cable with an electric motor. However, $L_{2}$ initially increases and then decreases and, hence, the rotation rate of the motor controlling this active cable has to be continuously adjusted as the pantograph is deployed by active cable 1 .

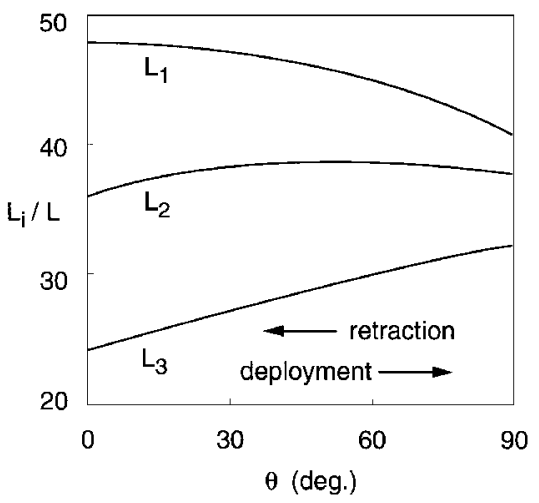

Fig. 6 Lengths of active cables during deployment. 
An alternative to the second active cable is shown in Fig. 5d. This active cable also fills the six gaps in the outer hoop and, hence, it is able to prestress the remaining hoop cables. The length of this cable increases monotonicallyduring deployment(Fig. 6), which makes it suitable to control retraction of the pantograph. Alternative arrangements of the active and passive cables have been identified by You. ${ }^{14}$

\section{Cable Network}

The ring pantographpresented in the preceding section provides a series of support points for the second component of the DMR. The cable network is required to be kinematically determinate $(m=0)$ and prestressable $(s>1)$ with a state of self-stress that involves tension in all cables. To simplify the task of setting up and maintaining the required state of prestress, it is desirable to have $s \approx 1$. Hence, according to Eq. (22), the total number of cables in the net should be approximately three times the number of joints. Also, of course, the shape of the network should approximate closely the required paraboloid.

In the tension truss, ${ }^{7}$ the cable network is triangulated and each joint is connected to six neighboring joints, thus forming a curved, six-way net that has the required number of cables per joint but, by itself, does not admit any states of self-stress. Hence, a series of tie cables are added, to connect each joint of the net to a backup cable network. The resulting network has $s \gg 1$ and, hence, although it is theoreticallypossible to set up a state of prestress involving tension everywhere, in practice this is quite difficult to achieve. This difficulty can be resolved by pursuing a global solution, rather than by adding extra cables to a net which is already rigid.

As we begin to investigate the topology of structures with the properties just listed, it is important that the joints of the cable network should be uniformly distributed, so that the same theoretical shape accuracy can be achieved everywhere. It is also important that it should be easy to change the joint density and the shape of the boundaries. Because we are dealing with shallow paraboloidal surfaces, these requirements can be met by projecting any standard plane-filling tessellation of regular polygons onto the required surface. Recall that there are only three plane-filling regular tessellations, based on triangles, squares, and hexagons; there are only eight semiregular plane-filling tessellations, based on combinations of equilateraltriangles, squares, hexagons, octagons, and dodecagons. In the plane, these are the only tessellations with uniformly distributed vertices.$^{15}$ For simplicity, we will describe two cable networks whose number of edge connection points is identical to the number of joints of the ring pantograph. More finely subdividednetworks of the same type can be obtained, if required, by increasing the number of polygons and without changing the ring pantograph.

\section{A. Double-Layer Network}

The pantograph structure provides 24 support points along its inner ring, on two separate layers. Hence, it is naturalto considerfirst double-layercable networks consisting of two similarly shaped nets connected by tie cables, where only one of the two nets, the primary net, is used to support the reflective mesh. Having considered all regular and semiregular tessellations as possible candidates for the two nets, the best solution is shown in Fig. 7. The primary net is a four-way semiregular tessellation of hexagons and triangles, whereas the secondary net is a three-way regular tessellation of hexagons. Note that the primary net has the higher joint density. Each joint of the secondarynet, e.g., joint 4 , is connectedby means of three cable ties to the nearest three joints of the primary net, i.e., joints 1 , 2,3 , and, thus, each joint of the primary net is supported by two cable ties. Each edge joint is connected to the edge frame by a single tie cable. This cable network has $j=66$ (respectively, 42 joints in the primary net and 24 in the secondary net) and $b=198$ (respectively, 72 bars in the primary net, 30 in the secondary net, 72 net-net ties, and 24 edge ties). Substituting these values into Eq. (22) gives

$$
m-s=0
$$

The same result is obtained for any network obtained by adding complete rings of hexagons and triangles along the edges of the primary and secondary nets just described.

The values of $m$ and $s$ are related to the rank of the equilibrium matrix of this cable network, and an analysis of this matrix ${ }^{11}$ shows

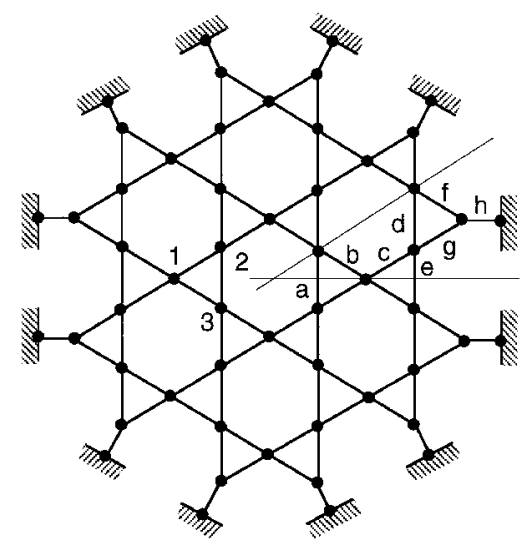

a) Primary net

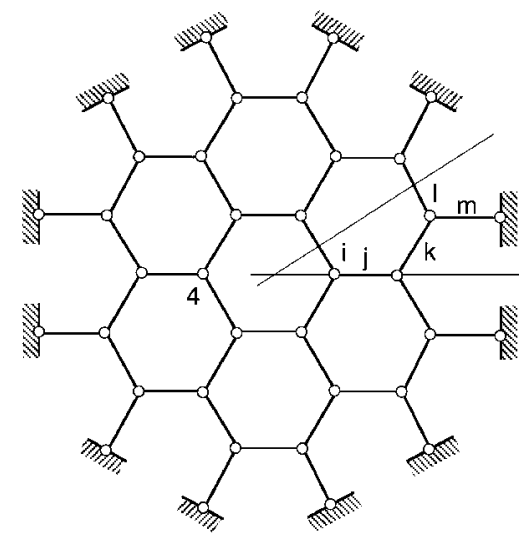

b) Secondary net

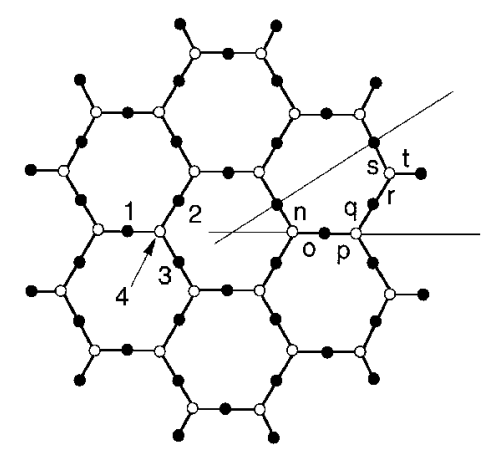

c) Cable ties

Fig. 7 Top views of double-layer cable network.

that $m=s=2$, regardless of the curvature of the two nets. The first state of self-stress has sixfold symmetry as well as reflection symmetry about six planes. All elements are in a state of tension, with the highest tension in the edge ties and the lowest in some of the net-net ties. The ratio between the tension in the edge ties of the primary net and those of the secondary net depends on the curvature of these two nets. The second state of self-stress involves alternate tension and compression in each pair of parallel edge ties, of much larger magnitude in the ties of the secondary net than in those of the primary net. It also involves large tensions and compressions in the tie members. Obviously, the first state of self-stress is the desirable one, in order to pretension the cable network.

The cable tensions in the network have been calculated for the particular case where the equations of the two nets are

$$
\begin{gathered}
Z_{p}=1.0811 \times \times^{10^{-4} R^{2}+126} \\
Z_{s}=-2.5409 \times{ }^{10^{-4} R^{2}}
\end{gathered}
$$

where $Z_{p}$ and $Z_{s}$ are the distance of the primary and secondary net from a reference plane, respectively, and $R$ is the radius within the reference plane, both measured in millimeters. Note that the primary net forms a paraboloid with a focal length of $2312 \mathrm{~mm}$. The results are given in Table 1 . Because of the symmetry of this 
Table 1 Prestress of cable networks

\begin{tabular}{|c|c|c|}
\hline Cable & $\begin{array}{c}\text { Tension } \\
\text { (double layer) }\end{array}$ & $\begin{array}{c}\text { Tension } \\
\text { (single layer) }\end{array}$ \\
\hline $\mathrm{a}$ & 31.56 & 17.49 (maximum) \\
\hline $\mathrm{b}$ & 31.62 & 10.58 \\
\hline $\mathrm{c}$ & 33.24 & 8.79 \\
\hline $\mathrm{d}$ & 33.68 & 5.48 \\
\hline e & 33.00 & 8.36 \\
\hline $\mathrm{f}$ & 33.85 & 5.62 \\
\hline $\mathrm{g}$ & 34.15 & 6.97 \\
\hline $\mathrm{h}$ & 60.65 (maximum) & 10.99 \\
\hline $\mathrm{i}$ & 30.09 & 3.95 \\
\hline $\mathrm{j}$ & 29.85 & 7.63 \\
\hline $\mathrm{k}$ & 28.71 & 1.78 \\
\hline 1 & 27.62 & 1.00 (minimum) \\
\hline $\mathrm{m}$ & 30.32 & 2.21 \\
\hline $\mathrm{n}$ & 2.74 & $\longrightarrow$ \\
\hline $\mathrm{o}$ & 4.50 & - \\
\hline $\mathrm{p}$ & 1.00 (minimum) & - \\
\hline $\mathrm{q}$ & 3.32 & - \\
\hline $\mathrm{r}$ & 1.32 & - \\
\hline s & 2.21 & - \\
\hline $\mathrm{t}$ & 2.94 & - \\
\hline
\end{tabular}

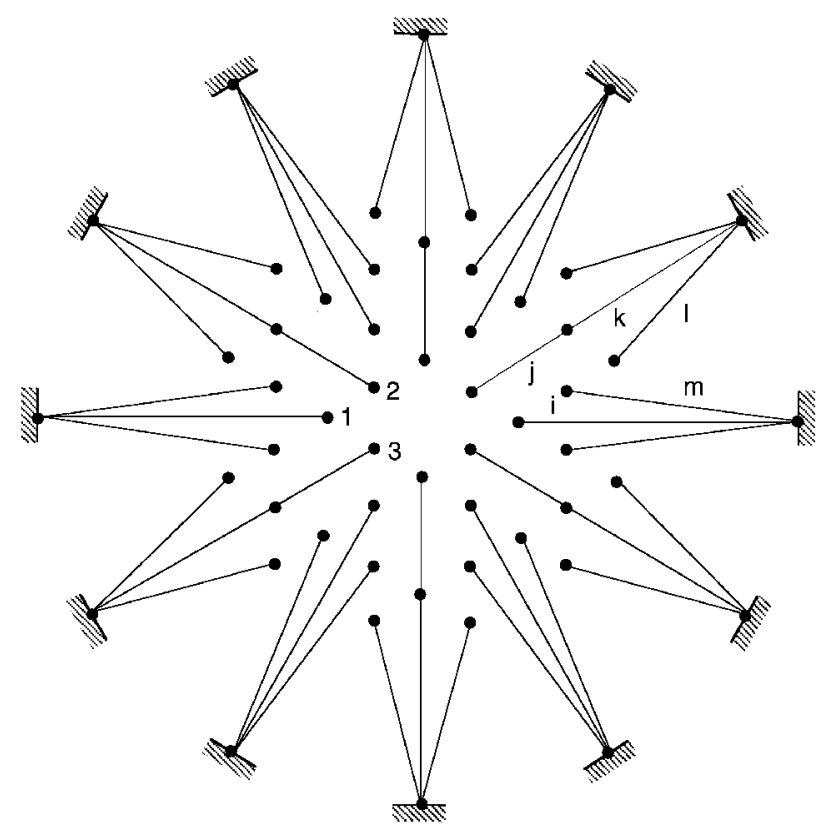

Fig. 8 Cable ties of single-layer cable network.

state of self-stress, all cable tensions can be obtained from the 20 values that have been listed. Note that, apart from the edge ties of the primary net, the tension distribution in the two nets is nearly uniform. However, the tensions in the tie cables are much lower, and there is the danger that these cables might become slack. Increasing the curvature of the secondarynet, which would not change the focal length of the reflector, tends to increase these tensions, but only by a small amount. It is likely that more significant improvements would be obtained if the joints of the two nets were allowed to move on their respective surfaces, but the required shape-and-stress optimization is yet to be attempted.

Assuming that the reflective flexible surface is a triangulated surface whose vertices coincide with the nodes of the primary net, the rms error of this surface would be $3.0 \mathrm{~mm}$. The $m=2$ inextensional mechanisms of this network correspond to small rotations of each net about the axis of the paraboloid. These mechanisms are stabilized when the network is prestressed and, indeed, they could be eliminated by adding a single tangential restraint to each net.

\section{B. Single-Layer Network}

An alternative to the double-layernetwork is obtained by removing the secondary net and connecting the cable ties directly to the lower joints of the outer ring of the pantograph structure (Fig. 8). The outer ring joints are used in order to avoid any compression.
The single-layernetwork has $j=42$ and $b=126$ ( 72 bars in the primary net plus 54 edge ties). Equation (21) gives

$$
m-s=0
$$

and a computer analysis of the equilibrium matrix shows that $m=s=2$. The two mechanisms are a rigid-body rotation of the entire network and a distortion of the hexagons in the net, respectively. These mechanisms can be eliminated by adding two more edge restraints.

For a net shaped according to the first equation in Eq. (27), the best combination of the two states of self-stress is given in Table 1 . In this solution the ratio between the maximum and minimum cable forces is 17.5 , much smaller than the double-layernetwork. Moreover, the maximum tension occurs in the cables of the central hexagon not in the edge ties and, hence, the load on the support structure is lower. The only disadvantage of the single-layernetwork concept is that some of its tie cables are considerably longer than those in the previous concept.

\section{Design of Test Model}

To verify the feasibility of the DMR concept described in the preceding sections, a physical model has been designed and made of a 12 -sided ring pantograph $(\ell=366 \mathrm{~mm}$ and $L=579 \mathrm{~mm})$ supporting a double-layer cable network. Further details are given in Table 2 .

In the fully deployed configuration $(\theta=90 \mathrm{deg})$, the outer and inner diameters of the ring pantograph are 3481 and $2201 \mathrm{~mm}$, respectively. In the fully folded configuration $(\theta=7.2 \mathrm{deg}$, due to joint interference), the outer diameter is reduced to $600 \mathrm{~mm}$, whereas the height of the ring increases to $1170 \mathrm{~mm}$. A further reduction of the packaged diameter could be obtained by redesigning the inner joints. The length of the first active cable (Fig. 5b) decreases from 27.765 to $23.722 \mathrm{~m}$ during deployment. Its total length variation is $4.043 \mathrm{~m}$. The length of the second active cable (Fig. 5c), increases from $20.844 \mathrm{~m}$ for $\theta=7.2 \mathrm{deg}$ to $22.484 \mathrm{~m}$ for $\theta=53.1 \mathrm{deg}$ and then decreases to $21.687 \mathrm{~m}$ for $\theta=90 \mathrm{deg}$. Thus, the maximum length variation is $1.640 \mathrm{~m}$. The lengths of these active cables are controlled by two dc motors with gearboxes, each turning a slender drum mounted alongside a rod of the pantograph (Fig. 9a), and the cable tension in the fully deployed configuration is controlled by setting a current cutoff on the power supply.

Each joint assembly acts as the connection point for four rods of the pantograph and one or more passive cables. Joints lying on the outer ring also support the active cables pulleys, whereas joints lying on the inner ring are also connected to the cable network. Each joint assembly consists of a block, with slots for inserting the rod connectors, and of a cover plate. The joint eccentricitiesare $\lambda=26.0$ $\mathrm{mm}$ (inner joints) and $\Lambda=41.1 \mathrm{~mm}$ (outer joints) (see Fig. 9b). Note that Eq. (21) is satisfied.

The deployment sequence of the model structure is shown in Fig. 1. Initially, the cable net is neatly folded in the middle of the pantograph. When the fully deployed configuration is reached, the two active cables pretension both the passive cables of the ring and the cable network simultaneously. Note that the model is supported by means of three steel ropes, which are approximately vertical when the model is fully folded and open out during deployment, thus applying gradually increasing lateral forces on the ring. The force in each hanging cable changes from $59.6 \mathrm{~N}$, in the folded configuration ( $\frac{1}{3}$ of the structural weight), to $64.9 \mathrm{~N}$ in the fully deployed configuration.

\section{Tests}

Two types of tests were carried out on the DMR model without the cable network: stiffness tests and deployment tests. Measurements were taken with a computer-controlled system of electronic

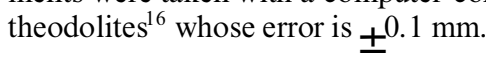

\section{A. Stiffness Tests}

The stiffness tests were carried out with the ring in its fully deployed and prestressed configuration. First, the lengths of all passive cables were adjusted to their nominal values, and the power supply to each electric motor was set to a voltage of $12 \mathrm{~V}$. The current 
Table 2 Properties of physical model

\begin{tabular}{|c|c|c|c|c|c|c|c|}
\hline \multirow[b]{3}{*}{ Components } & & & & \multicolumn{4}{|c|}{ Cables } \\
\hline & \multicolumn{3}{|c|}{ Rods } & \multicolumn{2}{|c|}{ Passive } & \multirow[b]{2}{*}{ Active } & \multirow[b]{2}{*}{ Network } \\
\hline & Inner & Near-radial & Outer & Near-radial & Outer & & \\
\hline Material & \multicolumn{3}{|c|}{ Al-alloy } & \multicolumn{2}{|c|}{ Kevlar $^{\circledR}$} & Steel & Kevlar \\
\hline Number & 24 & 48 & 24 & 24 & 18 & 2 & 198 \\
\hline Density, $\mathrm{kg} / \mathrm{m}^{3}$ & - & 2700 & - & \multicolumn{2}{|c|}{1440} & 7820 & 1440 \\
\hline Young's modulus, GN/m² & - & 69 & - & \multicolumn{2}{|c|}{60} & 200 & 60 \\
\hline Cross-sectional area, $\mathrm{mm}^{2}$ & - & 36.7 & - & \multicolumn{2}{|c|}{2.0} & 0.22 & 0.29 \\
\hline Length, mm & 732 & 945 & 1158 & 685 & 819 & Variable & $229-412$ \\
\hline
\end{tabular}

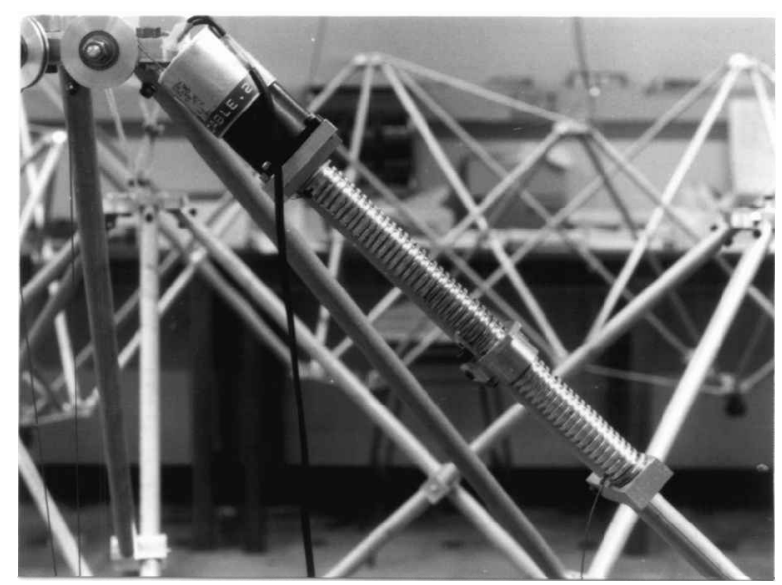

a) Drum

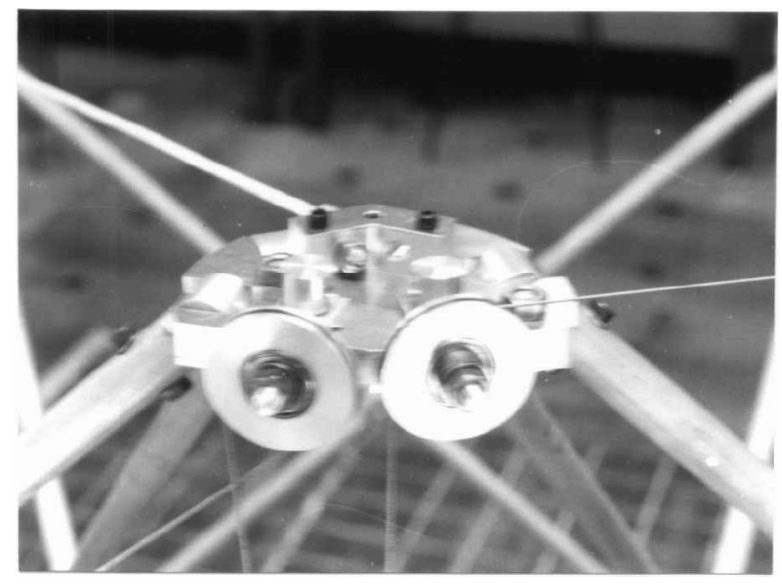

b) Outer ring joint

Fig. 9 Key components of test model.

cutoffs on the two motors were set to 0.5 and $0.15 \mathrm{~A}$, respectively, to achieve a tension ratio of about 2.551 [Eq. (24)] between active cables 1 and 2 . Then, the model was deployed and all cable tensions were measured with a tension meter. ${ }^{1}$ The measured tensions were $68 \mathrm{~N}$ in active cable 1, 27 $\mathrm{N}$ in active cable 2, and varying in the range $24-30 \mathrm{~N}$ in the passive cables. Note that, according to Sec. II.B, one would expect to find $26.6 \mathrm{~N}$ in active cable 2 and in all passive cables, for a tension of $68.0 \mathrm{~N}$ in active cable 1 . Once the model was fully deployed and prestressed, three different sets of four forces of equal magnitude were applied to the inner ring (Fig. 10a), in small increments. Because the ring was free to rotate as a rigid body about the suspension point, instead of measuring absolute displacements, four distance variations between opposite joints of the inner ring were measured (Fig. 10b). The measured distance variations are plotted vs the applied load in Fig. 11, together with the computed linear-elastic response of the ring. ${ }^{14}$

It is found that the behavior of the structure is almost perfectly linear for all load conditions, confirming that the imposed state of prestress has successfully eliminated hinge backlash. As in the triangular mast, ${ }^{1}$ the experimentalresults are generally in good agreement with the corresponding computational results. Note that the

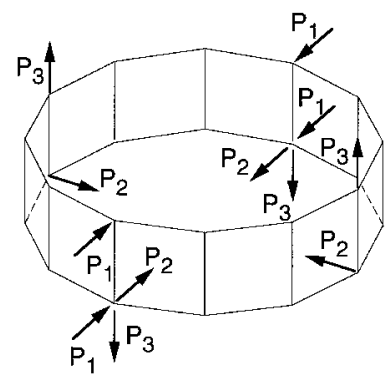

a) Three load cases

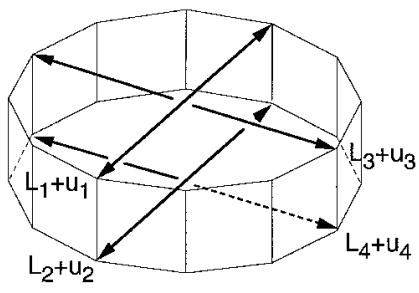

b) Four distances measured

Fig. 10 Stiffness tests.
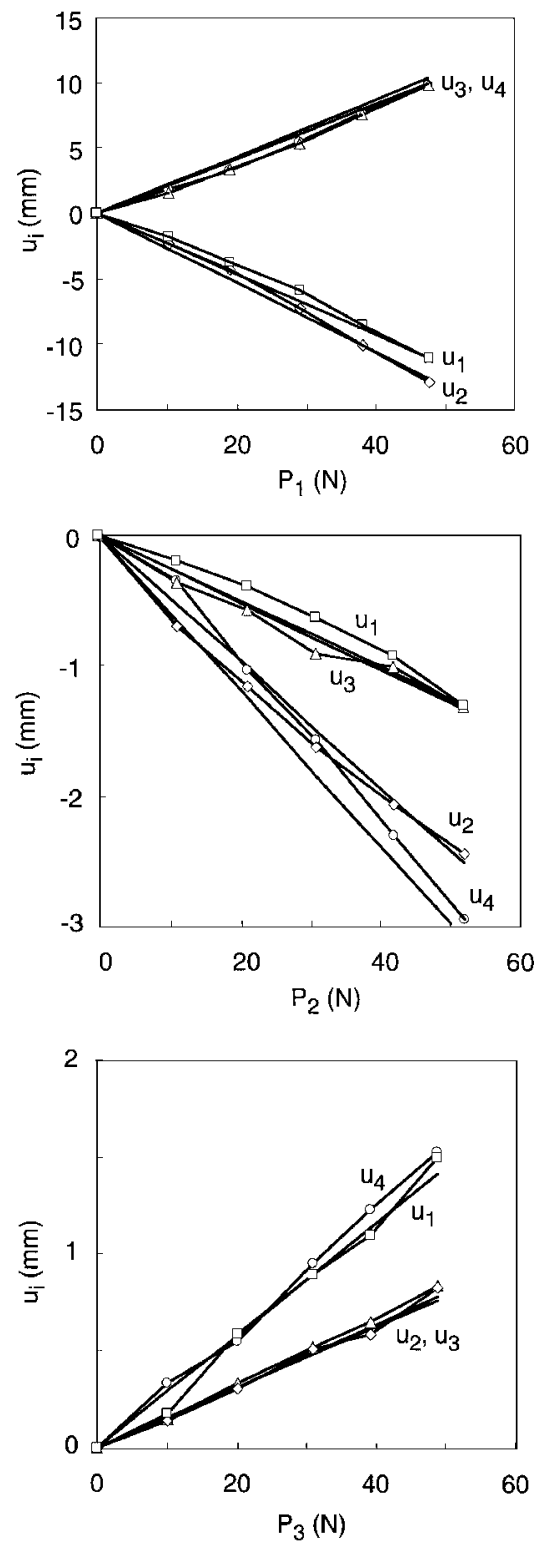

Fig. 11 Variation of joint distance vs load applied load. 


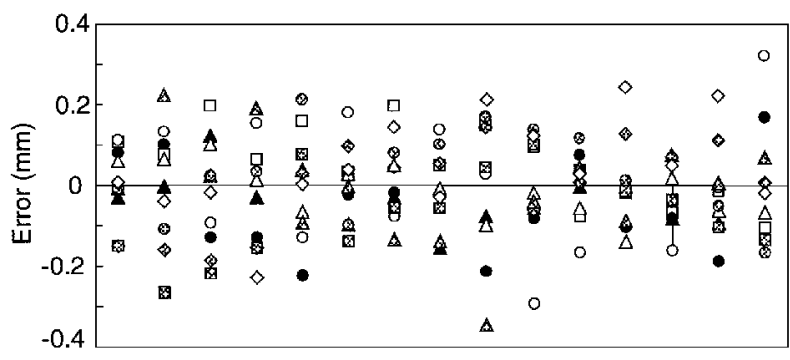

1-2 1-3 1-4 1-5 1-6 2-3 2-4 2-5 2-6 3-4 3-5 3-6 4-5 4-6 5-6

Distance (joint no - joint no)

Fig. 12 Results of deployment tests.

behavior of the structure is not perfectly symmetric, although the loads applied on it are symmetric, because of the lower-order symmetry of the hoop passive cables and of the second active cable.

Recall that in Sec. II.B it was noted that the pin-jointed model of the ring pantograph has an internal mechanism if the scissor hinge connecting each pair of rods is neglected. This mechanism is activated by the first load case, which, therefore, producessignificant bending and twisting of the rods and also larger deflections than the other load cases. The loads applied to the ring by the cable network do not activate this mechanism.

\section{B. Deployment Tests}

A series of fold-and-deploytests were carried out to measure how accurately the shape of the ring can be repeated. In each test the deployment process was terminatedautomaticallyby the current cutoff levels for the two motors, as explained in Sec. V.A, and the coordinates of alternate joints on the top side of the inner ring, six in total, were measured. The distances between these joints were then computed;Fig. 12 is a plot of the measured errors from the mean distance.

The maximum error range is $+0.3 \mathrm{~mm}$, approximately double the $x-y$ error range obtained from the $1.6-\mathrm{m}$-long triangular mast. ${ }^{1}$ The main source of shape error in these two pantographic deployable structures is manufacturing tolerance in the joints and, hence, the shape error might be expected to increase approximately linearly with the total number of joints. In fact, the shape error of the DMR ring appears to be rather better than this prediction. No detailed measurements of shape error and prestress of the cable network were taken during the course of this study, as any errors could be removed by the approach described in Ref. 17.

\section{Conclusion}

A new concept for deployablemesh reflectors has been presented. The first main component of the new concept is a deployable pantographic ring. Although a highly redundant system, during deployment this ring has an internal mechanism that permits synchronous deployment without any strain in the rods. The geometric conditions that have to be satisfied for an $n$-sided ring to fold without any strain have been investigated, and it has been shown that the ratio between the rod lengths is uniquely defined. A similar condition applies to the ratio between joint eccentricities. Active and passive cables are used to deploy and stiffen the pantograph. Several arrangements for the passive cables and the associated active cables have been found. These solutions ensure that all cables are in a state of uniform pretension when the structure is fully deployed.

The second main component of the new concept is a kinematically determinate cable network that is deployed and prestressed by the pantographic ring. Most existing cable nets are either kinematically indeterminate and therefore lack accuracy and stiffness or statically indeterminate with high redundancy, which makes prestressing extremely difficult. The two cable networks that have been proposed are kinematically determinate and have only two states of self-stress and, hence, they can be pretensioned by controlling the length of only two cables. They provide a series of regularly spaced support points for the reflective mesh or membrane of the reflector.
Hence, these networks overcome the difficulties usually associated with cable-supported reflective elements.

A 3.5-m-diam test model of the DMR has been made and tested. This model works well, thus demonstrating the validity of the proposed concept. Stiffness and deployment tests have shown that the concept is simple, reliable, and accurate.

The new reflector concept is suitable both for symmetric and offset configurations, as the shape of the cable network can be changed without a significant change in the state of prestress, and the tie cables along the edge of the net need not be symmetric. The concept is not tied to a particular type of subreflector/antenna feed support system, because it does not have a central mast. For example, in communication satellites it is likely that the feed will be mounted directly on the spacecraft bus, the reflector itself being mounted at the tip of a boom.

\section{Acknowledgments}

Financialsupportfrom the Engineeringand PhysicalSciencesResearch Council (Research Grant GR/F57113) is gratefully acknowledged. We are grateful to R. J. Denston for technical assistance.

\section{References}

${ }^{1}$ You, Z., and Pellegrino, S., "Cable-Stiffened Pantographic Deployable Structures Part 1: Triangular Mast," AIAA Journal, Vol. 34, No. 4, 1996, pp. 813-820.

${ }^{2}$ Reibaldi, G. C., "Antenna Mechanical Technologies Within ESA," Proceedings of 2nd ESA Workshop on Mechanical Technology for Antennas, European Space Agency, Noordwijk, The Netherlands, 1986, pp. 5-13.

${ }^{3}$ Freeland, R. E., "Survey of Deployable Antenna Concepts," Proceedings of Large Space Antenna Systems Technology-1982, NASA-CP-2269, Nov.-Dec. 1982, pp. 381-421.

${ }^{4}$ Roederer, A. G., and Rahmat-Samii, Y., "Unfurlable Satellite Antennas: A Review," Annales des Telecommunications, Vol. 44, No. 9, 10, 1989, pp. $475-488$.

${ }^{5}$ Allen, B. B., and Butler, D. H., "Hoop/Column Antenna Deployment Mechanism Overview," Proceedings of 19th Aerospace Mechanisms Symposium, NASA-CP-2371, May 1985 pp. 23-37.

${ }^{6}$ Harris Corp., "Development of the 15 Metre Diameter Hoop Column Antenna," NASA-CR-4038, 1986

${ }^{7}$ Miura, K., and Miyazaki, Y., "Concept of the Tension Truss Antenna," AIAA Journal, Vol. 28, No. 6, 1990, pp. 1098-1104.

${ }^{8}$ Pellegrino, S., and You, Z., "Foldable Ring Structures," Space Structures 4, edited by G. A. R. Parke and C. M. Howard, Vol. 1, Thomas Telford, London, 1993, pp. 783-792.

${ }^{9}$ Escrig, F., "Expandable Space Structures," International Journal of Space Structures, Vol. 1, 1985, pp. 79-91.

${ }^{10}$ Calladine, C. R., "Buckminster Fuller's Tensegrity Structures and Clerk Maxwell's Rules for the Construction of Stiff Frames," International Journal of Solids and Structures, Vol. 14, 1978, pp. 161-172.

${ }^{11}$ Pellegrino, S., "Structural Computations with the Singular Value Decomposition of the Equilibrium Matrix," International Journal of Solids and Structures, Vol. 30, No. 21, 1993, pp. 3025-3035.

${ }^{12}$ Kwan, A. S. K., and Pellegrino, S., "Prestressing a Space Structure," AIAA Journal, Vol. 31, No. 10, 1993, pp. 1961-1963.

${ }^{13}$ Kwan, A. S. K., and Pellegrino, S., "Matrix Formulation of MacroElements for Deployable Structures," Computers and Structures, Vol. 50, No. 2, 1994, pp. 237-254

${ }^{14}$ You, Z., "Deployable Structures for Masts and Reflector Antennas,' Ph.D. Dissertation, Dept. of Engineering, Univ. of Cambridge, Cambridge, England, UK, Aug. 1994.

${ }^{15}$ Gasson, P. C., Geometry of Spatial Forms, Ellis Horwood, Chichester, England, UK, 1983.

${ }^{16}$ Kwan, A. S. K., and You, Z., "User Guide to Theo3, an Industrial Measurement System Using Three Zeiss ETh2 Theodolites," Dept. of Engineering, Univ. of Cambridge, Rept. CUED/D-STRUCT/TR142, Cambridge, England, UK, 1993.

${ }^{17}$ Kawaguchi, K., Hangai, Y., Pellegrino, S., and Furuya, H., "Shape and Stress Control Analysis of Prestressed Truss Structures," Proceedings of the 5th International Conference on Adaptive Structures (Sendai, Japan), edited by J. Tani, E. J. Breitbach, B. K. Wada, M. Uchiyama, and S. Chonan, Technomic, Lancaster, PA, 1994, pp. 184-193. 